\title{
Conhecimento da equipe de enfermagem sobre neurointensivismo em Unidades de Terapia Intensiva
}

\author{
Knowledge of the nursing team on neurointensivism in \\ Intensive Care Units
}

\author{
Conocimiento del equipo de enfermería em \\ neurointensivismo en Unidades de Cuidado Intensivo
}
Regina Neves Ribeiro ${ }^{1}$, Ana Paula de Freitas Oliveira ${ }^{2}$, Jocélio Matos Amaral $^{3}$, Patrícia da Silva Pires ${ }^{4}$, Andressa Oliveira Santos ${ }^{1}$, Loren Scarlet da Silva Teixeira5, Ana Karina Lomanto Rocha6, Thaise Ferraz Santos $^{7}$

\begin{abstract}
1.Enfermeira. Residência em Urgência no Hospital Geral de Vitória da Conquista, Universidade Federal da Bahia (UFBA). Vitória da Conquista-BA, Brasil.

2.Enfermeira. Doutora em Enfermagem pela Faculdade de Ciências Médicas da Santa Casa de São Paulo. Professor Associado do Instituto Multidisciplinar em Saúde, Campus Anísio Teixeira, Universidade Federal da Bahia (UFBA). Vitória da Conquista-BA, Brasil.

3.Enfermeiro. Pós-graduado em UTI e residência em Urgência no Hospital Geral de Vitória da Conquista, Universidade Federal da Bahia (UFBA). Vitória da Conquista-BA, Brasil.

4.Enfermeira. Doutora em Enfermagem pela Universidade de São Paulo. Professor Associado do Instituto Multidisciplinar em Saúde, Campus Anísio Teixeira, Universidade Federal da Bahia (UFBA). Vitória da Conquista-BA, Brasil.

5.Enfermeira. Pós-graduada em cardiologia e Residência em Urgência no Hospital Geral de Vitória da Conquista, Universidade Federal da Bahia (UFBA). Vitória da Conquista-BA, Brasil.

6.Enfermeira. Mestre em Enfermagem pela Universidade Federal da Bahia. Preceptora da Residência multiprofissional SESAB/UFBA. Coordenadora da UTI 3 do Hospital Geral de Vitória da Conquista Bahia. Vitória da Conquista-BA, Brasil.

7.Enfermeira. Coordenadora da UTI 3 do Hospital Geral de Vitória da Conquista Bahia. Vitória da Conquista-BA, Brasil.
\end{abstract}

\section{Resumo}

Objetivo. Avaliar o conhecimento da equipe de enfermagem sobre neurointensivismo. Método. Trata-se de um estudo de caráter descritivo e exploratório com abordagem quantitativa realizada de março a abril de 2018, em duas unidades de terapia intensiva adulto. Utilizou-se um instrumento validado com dados demográficos e profissionais dos entrevistados e oito questões pertinentes ao conhecimento acerca do neurointensivismo. O Teste exato de Fisher foi usado para determinar diferenças nas respostas entre as categorias profissionais. Resultados. Responderam ao instrumento 61 profissionais de enfermagem, sendo 21 enfermeiros e 40 técnicos de enfermagem, $69 \%$ eram do sexo feminino. Os enfermeiros tiveram mais respostas corretas, que técnicos de enfermagem, porém, em nenhuma das questões esses profissionais apresentaram 100\% de acertos. Cuidados específicos da enfermagem para prevenir a elevação da PIC, ainda não são suficientemente conhecidos pelos profissionais, houve diferença estatisticamente significativa somente para o parâmetro de oximetria jugular SJO2 $(p<0,047)$ e estratégia de combate a hipertermia $(p<0,007)$. Conclusão. Essa pesquisa demonstra a necessidade de intervenção que contemple aquisição de conhecimentos teórico-práticos, aprimoramento para interpretação dos parâmetros monitorados e consequente adoção de condutas seguras no manejo do doente neurocrítico.

Unitermos. Unidades de Terapia Intensiva; Cuidados Intensivos; Hipertensão intracraniana; Conhecimento; Cuidados de Enfermagem; Monitoramento 


\begin{abstract}
Objective. To evaluate the knowledge of the nursing team about neurointensivism. Method. This is a descriptive and exploratory study with a quantitative approach carried out from March to April 2018, in two adult intensive care units. A validated instrument with demographic and professional data from the interviewees and eight questions relevant to the knowledge about neurointensivism was used. Fisher's exact test was used to determine differences in responses between professional categories. Results. 61 nursing professionals responded to the instrument, 21 nurses and 40 nursing technicians, 69\% were female. Nurses had more correct answers than nursing technicians, however, in none of the questions did these professionals show $100 \%$ correct answers. Specific nursing care to prevent elevation of the ICP, is not yet sufficiently known by professionals, there was a statistically significant difference only for the parameter of jugular oximetry $\mathrm{SJO} 2(\mathrm{p}<0.047)$ and strategy to combat hyperthermia $(p<0.007)$. Conclusion. this research demonstrates the need for intervention that includes the acquisition of theoretical and practical knowledge, improvement in the interpretation of monitored parameters and the consequent adoption of safe conducts in the management of neurocritical patients.
\end{abstract}

Keywords. Intensive Care Units; Intensive care; Intracranial hypertension; Knowledge; Nursing care; Monitoring

\title{
Resumen
}

Objetivo. Evaluar el conocimiento del equipo de enfermería sobre neurointensivismo. Método. Este es un estudio descriptivo y exploratorio con un enfoque cuantitativo llevado a cabo de marzo a abril de 2018, en dos unidades de cuidados intensivos para adultos. Se utilizó un instrumento validado con datos demográficos y profesionales de los entrevistados y ocho preguntas relevantes para el conocimiento sobre neurointensivismo. La prueba exacta de Fisher se utilizó para determinar las diferencias en las respuestas entre las categorías profesionales. Resultados. 61 profesionales de enfermería respondieron al instrumento, 21 enfermeras y 40 técnicos de enfermería, el $69 \%$ eran mujeres. Las enfermeras tenían más respuestas correctas que los técnicos de enfermería, sin embargo, en ninguna de las preguntas estos profesionales mostraron respuestas $100 \%$ correctas. La atención de enfermería específica para prevenir la elevación de la PIC, aún no es suficientemente conocida por los profesionales, hubo una diferencia estadísticamente significativa solo para el parámetro de oximetría yugular $\mathrm{SJO} 2(p<0.047)$ y la estrategia para combatir la hipertermia $(p<0.007)$. Conclusion. Esta investigación demuestra la necesidad de una intervención que incluya la adquisición de conocimientos teóricos y prácticos, la mejora en la interpretación de los parámetros monitoreados y la consecuente adopción de conductas seguras en el manejo de pacientes neurocríticos.

Palabras clave. Unidades de Cuidados Intensivos; Cuidados intensivos; Hipertensión intracraneal; Conocimiento; Cuidado de enfermeira; Supervisión

Trabalho realizado no Hospital Geral de Vitória da Conquista, Vitória da Conquista-BA, Brasil.

Endereço para correspondência: Regina N Ribeiro. R. da Misericórdia 533, Centro. CEP 45000-200. Vitória da Conquista-BA, Brasil. Email: reginaribeirobdo@hotmail.com

\section{INTRODUÇÃO}

\author{
A Unidade de Terapia intensiva (UTI) se destina ao
} tratamento de pacientes em estado crítico, assistindo-os de forma ininterrupta e com recursos específicos para 0 
restabelecimento de suas funções orgânicas ${ }^{1,2}$. Estas unidades possuem espaço físico específico, recursos humanos especializados e instrumentais tecnológicos avançados que permitem uma assistência adequada a pacientes críticos, incluindo aqueles com alterações neurológicas ${ }^{3}$.

Entre os pacientes que são admitidos na UTI com problemas neurológicos, destacam-se aqueles com lesão cerebral grave, e esta se encontra associada a diversas condições etiológicas, como: traumatismo cranioencefálico grave (TCE), acidente vascular encefálico isquêmico/hemorrágico, trauma raquimedulares (TRM), estado epiléptico, miastenia grave (MG) bem como o caso de pacientes neurocirúrgicos de alto risco na fase pósoperatória ${ }^{4-6}$.

Estudos mostram que os elevados coeficientes de sequelas motoras, psicológicas, comportamentais e cognitivas decorrentes do TCE ou por outro evento cerebral, quando não são advindas do próprio mecanismo traumático, surgem a partir de diversos fatores secundários que estão envolvidos na evolução do quadro ${ }^{7-9}$.

Assim, é imprescindível a detecção precoce de alterações hemodinâmicas e neurológicas, com foco no controle rigoroso da hipotensão e hipóxia cerebral ${ }^{10,11}$. Além disso, as diretrizes baseadas em evidências recomendam acompanhamento e avaliação precoce do estado neurológico, tamanho e reação pupilar, avaliação das 
funções motoras, convulsões e outros sinais de hipertensão intracraniana ou herniação cerebral ${ }^{11,12}$.

O monitoramento dos parâmetros de pressão intracraniana (PIC), fluxo sanguíneo cerebral (FSC), pressão arterial media (PAM), pressão de perfusão cerebral (PPC), extração cerebral de oxigênio (ECO2), pressão venosa central e saturação venosa jugular (SJO2) são importantes por fornecer informações úteis para o manejo ideal em pacientes neurocríticos ${ }^{13}$.

Neste contexto, a equipe de enfermagem deve buscar conhecimento com base em evidências científicas atualizadas e bem recomendadas, tendo em vista que 0 atendimento ao paciente neurocrítico demanda cuidados específicos, pressupondo o desenvolvimento e a aplicação criteriosa de conhecimentos teóricos pertinentes e habilidades de raciocínio para oferta de uma assistência segura e com intervenções assertivas ${ }^{14,15}$.

Diante disso, a realização deste estudo se justifica pela necessidade de avaliar se a equipe de enfermagem possui domínio suficiente para assistir adequadamente o paciente neurocrítico, uma vez que esta se constitui elemento essencial para a avaliação neurológica, tendo em vista a sua constante permanência perante 0 paciente grave. Além disso, existem evidências ${ }^{11,16}$ substanciais de que 0 tratamento desses pacientes em centros com manejo controlado por protocolos contribui para a diminuição de complicações e melhores resultados. 
O objetivo deste estudo foi avaliar o conhecimento da equipe de enfermagem sobre neurointensivismo.

\section{MÉTODO}

\section{Amostra}

Estudo de caráter descritivo e exploratório com abordagem quantitativa, desenvolvido em duas unidades de terapia intensiva tipo adulto, que possuem 19 leitos de atendimento geral, localizadas em um hospital geral de Vitória da Conquista (BA), Brasil. A coleta dos dados foi realizada no período de março a abril de 2018.

As unidades de Terapia Intensiva (UTI) até o momento da realização da pesquisa não possuiam um protocolo gerenciado para o doente neurocrítico. Embora, dispusesse de capacidade para monitorar continuamente a saturação de oxigênio, o ritmo cardíaco, a frequência cardíaca e pressão arterial (invasiva e não invasiva) não possuiam dispositivos fundamentais como capnográfo em todos os monitores das UTIs. A derivação ventricular externa era utilizada somente para drenagem sem monitoramento de PIC, saturação venosa jugular também não era comumente monitorada.

A realização da pesquisa obedeceu às normas éticas e legais vigentes e foi aprovada pelo Comitê de Ética em Pesquisa do Instituto Multidisciplinar em Saúde-Campus Anísio Teixeira mediante o CAAE 82571818.1.0000.5556.

Foram incluídos no estudo enfermeiros e técnicos de enfermagem que trabalhavam na UTI com no mínimo 06 
meses de experiência. Foram excluídos aqueles que usufruíam de férias ou estavam em afastamento médico nos meses de coleta.

\section{Procedimento}

A coleta de dados foi realizada de acordo com a disponibilidade de cada profissional, nos períodos matutino, vespertino e noturno. O preenchimento ocorreu de forma individual, sem acesso a fontes para consultas sob observação dos pesquisadores.

O questionário utilizado foi adaptado do estudo anterior $^{17}$ mediante autorização dos autores, composto por dados sócios demográficos (idade, sexo, tempo de serviço, cargo que ocupa) e oito questões estruturadas sobre o neurointensivismo.

Dentre as oito questões de múltipla escolha do questionário, em duas foi permitido marcar mais de uma opção como resposta, e seis eram de respostas únicas. Os questionários consistiram em itens para avaliar 0 conhecimento dos profissionais de enfermagem sobre parâmetros hemodinâmicos que devem ser observados no doente neurocrítico, cuidados de enfermagem durante e após a manipulação do paciente com hipertensão intracraniana (HIC) e os fatores e procedimentos que podem contribuir para elevação da PIC.

Os questionários incompletos não foram incluídos para análise. 


\section{Análise Estatística}

Todas as informações obtidas foram codificadas e inseridas em um banco de dados do programa Excel $2010 \AA$ e posteriormente calculadas frequências simples, além de percentuais para as variáveis categóricas. A análise estatística foi realizada com ajuda do programa SPSS (Statistical Package For Social Science for Windows), versão 25.0. O Teste exato de Fisher foi utilizado para testar se as respostas diferiam entre profissionais de enfermagem por nível de formação (enfermeiros versus técnicos de enfermagem). Valor de $p<0,05$ foi considerado significante.

\section{RESULTADOS}

Participaram do estudo 61 profissionais de enfermagem que atuavam nas UTI's, sendo 21 enfermeiros (34,4\%) e 40 técnicos de enfermagem (65,6\%). De modo geral, o sexo feminino foi predominante $(68,85 \%)$ com média de idade de $38,4 \pm 6,31$ anos e com tempo médio de serviço entre as

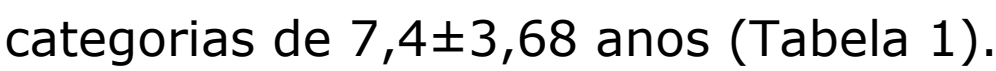

Com relação à participação em atividades educativas sobre neurointensivismo, (72\%) dos profissionais de enfermagem tinham realizado previamente algum treinamento dentro da instituição. 
Tabela 1. Dados demográficos da amostra $(\mathrm{N}=61)$.

\begin{tabular}{lccc}
\hline & Enfermeiros & Técnicos & Total \\
\hline Sexo - n(\%) & $14(67)$ & $28(70)$ & $42(69)$ \\
Feminino & $07(33)$ & $12(30)$ & $19(31)$ \\
Masculino & $37,36 \pm 5,6$ & $38,8 \pm 3,7$ & $38,36 \pm 6,31$ \\
Idade - média \pm desvio padrão & $6,9 \pm 3,7$ & $7,65 \pm 3,68$ & $7,38 \pm 3,68$ \\
Tempo de serviço (anos) - & & & \\
média \pm desvio padrão & & & \\
\hline
\end{tabular}

No que se refere à avaliação do conhecimento dos profissionais sobre quais parâmetros hemodinâmicos devem ser observados no doente neurocrítico, os enfermeiros apresentaram porcentagem de acertos maior que os técnicos de enfermagem em todos os parâmetros avaliados, entretanto, a SJO2 foi o único com diferença estatística significante ( $p=0,047$; Tabela 2$)$.

Embora $11 \quad(52,38 \%)$ dos enfermeiros tenha considerado a $\mathrm{SJO} 2$ como um parâmetro importante que deve ser avaliado, quando questionados sobre as indicações da coleta de sangue para verificação da saturação venosa cerebral, a porcentagem de acerto foi relativamente baixa, apenas $6(28,57 \%)$ para enfermeiros e 14 (35\%) para técnicos (Tabela 2).

Ainda ao serem indagados se conheciam os valores de normalidade dos parâmetros hemodinâmicos, 14(67\%) dos enfermeiros e $21(52 \%)$ dos técnicos responderam que possuíam conhecimento dos valores de referência.

Já em relação à periodicidade da avaliação e registro de PPC e de outros parâmetros adicionais que devem ser anotados, o percentual de acertos entre as categorias ficou abaixo dos (50\%). Ao mesmo tempo, quanto ao 
conhecimento acerca da realização do cálculo de PPC, somente $26(42,6 \%)$ dos profissionais obtiveram acerto (Tabela 2).

Tabela 2. Resultado de frequências e porcentagens corretas sobre parâmetros hemodinâmicos que devem ser observados no doente neurocrítico.

\begin{tabular}{lcccc}
\hline & $\begin{array}{c}\text { Enfermeiros } \\
(\mathbf{N = 2 1})\end{array}$ & $\begin{array}{c}\text { Técnicos } \\
(\mathbf{N = 4 0 )}\end{array}$ & $\begin{array}{c}\text { Total } \\
(\mathbf{N = 6 1 )}\end{array}$ & Valor de P \\
\hline 1. Parâmetros a serem & & & & \\
observados na HIC* & & & & \\
PPC & $14(67)$ & $16(40)$ & $30(49,1)$ & 0,062 \\
PAM & $20(95)$ & $31(77,5)$ & $51(83,6)$ & 0,142 \\
PVC & $13(62)$ & $16(40)$ & $29(47,5)$ & 0,116 \\
ECO2 & $12(57,14)$ & $15(37,5)$ & $27(44,3)$ & 0,179 \\
SJO2 & $11(52,38)$ & $10(25)$ & $21(34,4)$ & $<\mathbf{0 , 0 4 7}$ \\
PIC & $20(95)$ & $33(82,5)$ & $53(86,9)$ & 0,243 \\
2. Periodicidade dos & $10(47)$ & $16(40)$ & $26(42,62)$ & 0,596 \\
registros & & & & \\
3. Cálculo matemático de & $12(57,14)$ & $14(35)$ & $26(42,62)$ & 0,111 \\
PPC & & & & \\
4. Conhecimento sobre o & $6(28,57)$ & $14(35)$ & $20(32,78)$ & 0,775 \\
cateter bulbo jugular & & & &
\end{tabular}

* $\overline{\text { Respostas múltiplas; HIC - hipertensão intracraniana; PPC - pressão de perfusão cerebral; PAM - pressão }}$ arterial média; PVC - pressão venosa central; ECO2 - extração cerebral de oxigênio; SJO2 - Saturação venosa jugular; PIC - pressão intracraniana. Resultado expressos por n (\%). Teste de Fisher.

As medidas gerais quanto aos cuidados de enfermagem durante e após a manipulação do paciente com HIC (elevação da cabeceira do leito a 30\%, alinhamento mentoesternal, checagem do sistema de monitorização da PIC, prevenção de tração ou compressão do sistema de drenagem na presença de derivação ventricular externa, checagem do zero do sistema de drenagem) e procedimento de aspiração endotraqueal (10 a 15 segundos com prévia hiperventilação e aumento da FIO2) tiveram baixo percentual de acertos (menos de 50\%; Tabela 3). 
No tocante às estratégias de combate a hipertermia viu-se que os enfermeiros obtiveram maior porcentagem de acertos quando comparados aos técnicos, com diferença estatisticamente significativa $(p=0,007)$. Cabe destacar que a porcentagem de acertos dos técnicos de enfermagem foi consideravelmente baixa (Tabela 3 ).

Tabela 3. Distribuição amostra, segundo categoria profissional, de respostas certas e erradas sobre os cuidados de enfermagem na hipertensão intracraniana.

\begin{tabular}{|c|c|c|c|c|c|c|c|}
\hline & \multicolumn{2}{|c|}{$\begin{array}{c}\text { Enfermeiros } \\
(\mathbf{N}=\mathbf{2 1})\end{array}$} & \multicolumn{2}{|c|}{ Técnicos $(\mathrm{N}=40)$} & \multicolumn{2}{|c|}{ Total $(\mathrm{N}=61)$} & \multirow[t]{2}{*}{$\begin{array}{l}\text { Valor } \\
\text { de } p^{*}\end{array}$} \\
\hline & Acertos & Erros & Acertos & Erros & Acertos & Erros & \\
\hline $\begin{array}{l}\text { 5. Manipulação } \\
\text { do paciente } \\
\text { com HIC }\end{array}$ & $5(23,81)$ & $16(76,19)$ & $9(22,5)$ & $31(77,5)$ & $14(22,95)$ & $47(77,04)$ & 1,000 \\
\hline $\begin{array}{l}\text { 6. Aspiração } \\
\text { do tubo } \\
\text { traqueal na } \\
\text { HIC }\end{array}$ & $9(42,86)$ & $12(57,14)$ & $14(35)$ & $26(65)$ & $23(37,7)$ & $38(62,29)$ & 0,587 \\
\hline $\begin{array}{l}\text { 7. Estratégias } \\
\text { de combate a } \\
\text { hipertermia }\end{array}$ & $14(66,67)$ & $7(33,33)$ & $12(30)$ & $28(70)$ & $26(42,62)$ & $35(57,37)$ & 0,007 \\
\hline
\end{tabular}

A equipe de enfermagem de modo geral reconheceu, com percentual de acertos acima de 70\%, diversos fatores e procedimentos que elevam a PIC (aspiração, dor, sedação inadequada, compressão do tubo endotraqueal), no entanto, quanto à $\mathrm{PPC}<60 \mathrm{mmHg}$ e os parâmetros gasométricos de saturação periférica, pressão parcial de gás carbônico (os quais devem ser monitorizados e valores alvos atingidos, mantendo em níveis adequado hematócrito e hemoglobina) o estudo concluiu que mais da metade dos profissionais, de 
ambas as categorias, não detém conhecimento sobre esses valores gasométricos (Tabela 4 ).

Tabela 4. Distribuição amostral, por categoria profissional, de acertos relacionados a fatores relacionados à elevação da PIC.

\begin{tabular}{|c|c|c|c|c|}
\hline & $\begin{array}{l}\text { Enfermeiros } \\
\quad(N=21)\end{array}$ & $\begin{array}{l}\text { Técnicos } \\
(N=40)\end{array}$ & Total $(\mathrm{N}=61)$ & $\begin{array}{l}\text { Valor de } \\
\text { P** }\end{array}$ \\
\hline \multicolumn{5}{|l|}{$\begin{array}{l}\text { 8. Fatores e } \\
\text { procedimentos que } \\
\text { contribuem para } \\
\text { elevação da PIC* }\end{array}$} \\
\hline Aspiração & $20(95)$ & $32(80)$ & $52(85,24)$ & 0,145 \\
\hline Dor & $16(76,19)$ & $31(77,5)$ & $47(77,04)$ & 1,000 \\
\hline Sedação inadequada & $18(85,71)$ & $28(70)$ & $46(75,40)$ & 0,222 \\
\hline Compressão do TOT & $13(61,9)$ & $22(55,0)$ & $35(57,37)$ & 0,183 \\
\hline $\mathrm{PAM}<80 \mathrm{mmhg}$ & $10(47,62)$ & $21(52,5)$ & $31(50,81)$ & 0,791 \\
\hline $\mathrm{PPC}<60 \mathrm{mmhg}$ & $9(42,86)$ & $18(45)$ & $27(44,26)$ & 1,000 \\
\hline $\mathrm{SAO} 2<90 \%$ & $11(52,38)$ & $21(52,5)$ & $32(52,45)$ & 1,000 \\
\hline $\mathrm{PACO} 2<25 \%$ & $7(33)$ & $15(37,5)$ & $22(36)$ & 0,786 \\
\hline $\mathrm{HTM}<30 \mathrm{mg} / \mathrm{dl}$ & $8(38,1)$ & $13(32,5)$ & $21(34,42)$ & 0,778 \\
\hline $\mathrm{HB}<8,5$ & $6(28,57)$ & $15(37,5)$ & $21(34,42)$ & 0,577 \\
\hline
\end{tabular}

\section{DISCUSSÃO}

Dentre os principais achados deste estudo observou-se que nenhuma das categorias estudadas obteve $100 \%$ de acertos das questões, demonstrando assim lacunas de conhecimento neurointensivo num cenário que exige práticas seguras e competência técnica-científica.

Todavia, houve maior percentual de acertos de enfermeiros que de técnicos, entretanto sem diferença significante entre as categorias, na maioria dos itens analisados. Este resultado permite se questionar sobre 0 
nível de conhecimento dos enfermeiros que teoricamente devesse apresentar maior conhecimento teórico. Portanto, esperava-se que a diferença de acertos entre as categorias fosse maior.

Além disso, observou-se que o número de respostas corretas variou de 23,8 a 95\%, com média de acertos dos enfermeiros consideravelmente menor quando comparado a um estudo semelhante realizado em duas unidades de terapia intensiva para adultos de hospitais paranaenses ${ }^{17}$. Estudo realizado na Tailândia com 34 enfermeiros que atuavam em unidade de emergência mostrou que o número de respostas corretas variou de 33,3 a 95,2\%, com conhecimento e práticas de cuidado dos enfermeiros tailandeses para pacientes neurocrítico ${ }^{18}$.

Outro achado importante do estudo foi que maior parte dos técnicos de enfermagem não detêm conhecimento básico para reconhecimento da maioria dos parâmetros que devem ser observados no doente neurocrítico, embora afirmassem conhecer os valores de normalidade desses parâmetros. Isto é preocupante, visto que todos os parâmetros são importantes e estão intimamente relacionados à piora clínica e a elevação da PIC ${ }^{18,19}$. A monitorização desses parâmetros é imprescindível por permitir uma abordagem precoce a fim de evitar mais danos neurológicos e consequentes lesões secundárias ${ }^{20}$.

Entretanto, o baixo número de acertos pode estar relacionado ao fato das unidades em que a pesquisa foi realizada não utilizarem alguns desses parâmetros para 
monitorização, o que pode corroborar para tal lacuna de conhecimento entre os profissionais.

Por outro lado, a diferença significante de acertos referente à oximetria jugular para os enfermeiros pode estar relacionada por ser um parâmetro de alta complexidade, comumente não abordada nas formações de nível técnico. Em contrapartida no que diz respeito à indicação da coleta de sangue do cateter bulbo jugular o estudo observou que os enfermeiros não possuíam conhecimento das indicações. No entanto, vale ressaltar que a unidade não utiliza esse dispositivo de forma rotineira.

Sabe-se que a oximetria jugular permite avaliar as condições de oxigenação cerebral em pacientes neurocríticos, ao determinar se há equilíbrio entre a perfusão cerebral e o metabolismo cerebral ${ }^{16}$. Resultados de oximetria jugular inferiores a $55 \%$ sugerem situações isquêmicas, causada por hipoperfusão cerebral e resultados superiores a $75 \%$, tem como causa o edema cerebral ${ }^{16,21-23}$.

Alguns cuidados devem ser tomados como manter 0 lúmen do cateter da $\mathrm{SvjO}_{2}$ com solução salina a 0,9\%, não administrar drogas e utilizar o cateter apenas para monitorização e coletas para amostra de sangue ${ }^{9}$.

Em relação ao registro dos parâmetros hemodinâmicos, a equipe de enfermagem deve saber associar os valores de PAM e PIC que são determinantes da PPC. Pouco mais da metade ( $57 \%$ ) dos enfermeiros reconheceu essa associação, resultado esse inferior quando comparado com outro estudo similar ${ }^{17}$ o que pode denotar pouco conhecimento quanto ao 
cálculo necessário para controle de perfusão do Sistema Nervoso Central (SNC).

A maioria dos profissionais considerou que os registros de PIC e PPC devem ser documentados de $2 / 2$ horas. Porém, os guidelines recomendam que os registros devam ser de 1/1 hora até estabilização e de $2 / 2$ horas, se PIC estável; além disso, a monitorização se inicia por um exame neurológico sucinto no qual se avaliam sinais que indicam compressão do tronco ou dos nervos cranianos. Deve ser avaliado e registrado o nível de consciência o tamanho e a simetria das pupilas, bem como a sua reação à luz, balanço hídrico, ECG, sedação e débito urinário4,22.

Destaca-se neste estudo, que enfermeiros e técnicos de enfermagem demonstraram conhecimento insuficiente para os cuidados na manipulação do paciente com HIC: apenas $23 \%$ do total de profissionais reconhecem os cuidados. Esse fato é bastante temerário ao considerar que cuidados inadequados na manipulação do paciente neurocritico podem contribuir para um desfecho negativo do paciente ${ }^{4,22}$.

Cabe destacar ainda que, na UTI, a equipe de enfermagem é quem manipula o paciente e/ou auxilia os demais profissionais, portanto, a deficiência de conhecimento quanto à manipulação do paciente neurocrítico, implica diretamente na falta de sistematização adequada da assistência de enfermagem, como a construção de diagnósticos de enfermagem relacionados a $\mathrm{HIC}^{23}$ (ex: capacidade adaptativa intracraniana diminuída). Isso, 
consequentemente denota possíveis falhas nas intervenções de enfermagem neste contexto.

Em relação à aspiração do tubo endotraqual restritamente indicada, já que o próprio procedimento em si causa hipóxia e a aspiração no paciente crítico deflagra o reflexo de tosse que também eleva a PIC, não houve diferença significante entre as categorias. Contudo, questão como esta deveria ter apresentado maior percentual de acertos entre os enfermeiros, já que conforme dispoe a resolução N0557/2017 do Conselho Federal de Enfermagem (COFEN), a aspiração de vias aéreas em pacientes graves, submetidos à intubação orotraqueal é privativo dos enfermeiros ${ }^{24}$.

Existem evidências de elevação significativa dos parâmetros fisiologicos durante a aspiração traqueal e a dor foi significativamente mais intensa durante tal procedimento o que contribui para elevação da $\mathrm{PIC}^{25}$. Nesse sentido é válido destacar a importância de capacitações acerca das técnicas corretas de realização desse procedimento no doente neurocrítico.

No que diz respeito às estratégias de combate a hipertermia, houve diferença significante entre as categorias profissionais porque a média de acertos dos técnicos de enfermagem foi consideravelmente baixa. O fato de $70 \%$ dos técnicos não saber re/conhecer as estratégias de combate é um dado preocupante já que é uma função inerente dessa categoria a verificação da temperatura corporal. 
Sabe-se que a hipertermia aumenta o metabolismo cerebral e é causa importante de lesão neuronal cerebral ${ }^{26}$. A literatura aponta que devido à instabilidade hemodinâmica que pacientes críticos podem apresentar o combate a hipertermia deve ser agressivo, de forma que o paciente receba antitérmico e medidas gerais de resfriamento para manter temperatura corporal $\left(<37,5^{\circ} \mathrm{C}\right)$ e assim evitar possíveis lesões cerebrais secundárias ${ }^{9,27}$.

Quanto aos fatores e procedimentos que elevam a PIC os itens (aspiração, compressão pelos cadarços de fixação do tubo endotraqueal, sedação inadequada e dor) obtiveram maior frequência de acertos entre enfermeiros e técnicos. Sabe-se que todos esses fatores aumentam a demanda metabólica cerebral e elevam a PIC com consequente injuria cerebral $^{4,11,12}$. No entanto, $\mathrm{PPC}<60 \mathrm{mmHg}$ e dados gasométricos que também contribuem para elevação da PIC houve um baixo número de acertos por parte dos profissionais. O que pode comprometer a qualidade da assistência ao doente neurocrítico.

Nesse sentido é importante enfatizar que dentre as intervenções de enfermagem na HIC, avaliar gasometria arterial também é função do enfermeiro, que não deve se limitar apenas a coletar e interpretar o resultado como algo realizado de forma isolada. O enfermeiro deve em conjunto com a equipe multidisciplinar elaborar plano de cuidados e estratégias terapêuticas, de forma a colaborar para a recuperação do quadro clínico do doente neurocrítico ${ }^{27}$. 
Conforme a Resolução do $\operatorname{Cofen}^{28}$ no. 390/2011 o enfermeiro é responsável pela realização de cuidados de maior complexidade técnica e, portanto, é de fundamental importância que o enfermeiro saiba detectar, monitorar e estabelecer condutas de enfermagem frente aos distúrbios do equilíbrio acidobásico e de oxigenação ${ }^{23,29}$.

É importante ressaltar que estudos que abordam a assistência de enfermagem ao paciente neurocrítico são escassos. Contudo, o conhecimento sobre temas recorrentes da neurociência pelos profissionais de saúde possibilita a implantação de medidas que visam elaboração de protocolos de tratamento para redução da morbidade e da mortalidade.

Os resultados do presente estudo apontam um déficit de conhecimento quanto ao manejo do doente neurocrítico, principalmente entre os técnicos de enfermagem. Além disso, há necessidade de maior conhecimento neurointensivo e de cuidados de enfermagem mais precisos na HIC, já que o enfermeiro é peça chave na condução de protocolos dentro da UTI, sendo o responsável pelo planejamento da assistência, efetuando intervenções junto a equipe sob sua supervisão através de avaliação diária30,31.

Quanto às limitações do estudo, destaca-se o número de profissionais estudados e o número de Unidades (duas) que não permite a generalização dos dados. 


\section{CONCLUSÃO}

Verificou-se que os enfermeiros tiveram mais respostas corretas, que técnicos de enfermagem, porém, em nenhuma das questões esses profissionais apresentaram 100\% de acertos. Cuidados específicos da enfermagem para prevenir a elevação da PIC, ainda não são suficientemente conhecidos.

De fato, os resultados apontados no estudo sugerem lacunas de conhecimento acerca do manejo do doente neurocrítico, visto que $o$ cuidado em enfermagem neurológica demanda um amplo conhecimento por parte dos profissionais que prestam à assistência.

Portanto, o estudo pode contribuir para visualizar a realidade da abordagem e da atuação dos profissionais de enfermagem, influenciar um movimento de sensibilização e treinamento, bem como a necessidade de estabelecimento de protocolos baseados em evidências.

\section{REFERÊNCIAS}

1.Stocchetti N, Taccone FS, Citerio G, Pepe PE, Le Roux PD, Oddo M, et al. Neuroprotection in acute brain injury: an up-to-date review. Crit Care 2015;19:186. http://dx.doi.org/10.1186/s13054-015-0887-8

2.Stocchetti N, Carbonara M, Citerio G, Ercole A, Skrifvars MB, Smielewski $\mathrm{P}$, et al. Severe traumatic brain injury: targeted management in the intensive care unit. Lancet Neurol 2017;16:45264. https://doi.org/10.1016/S1474-44422(17)30118-7

3.Marinho RC, Souza RD, Lima DS. Pós-operatório de cirurgia neurológica. In: Viana RA, Torre M. Enfermagem em Terapia intensiva: praticas integrativa. Barueri: Manole; 2017, p807-16.

4.Oddo M, Crippa IA, Mehta S, Menon D, Payen, JF, Taccone FS, et al. Optimizing sedation in patients with acute brain injury. Crit Care 2016;20:128. http://dx.doi.org/10.1186/s13054-016-1294-5

5.Ministério da Saúde. Diretrizes de Atenção à Reabilitação da Pessoa com Traumatismo Cranioencefálico. Brasília: Ministério da Saúde, 
2015.

http://bvsms.saude.gov.br/bvs/publicacoes/diretrizes atencao reabili tacao pessoa traumatisco cranioencefalico.pdf

6. Magalhães ALG, Souza LC, Faleiro RM, Teixeira AL, Miranda AS. Epidemiologia do traumatismo cranioencefálico no Brasil. Rev Bras Neurol 2017;53:15-22.

https://revistas.ufrj.br/index.php/rbn/article/view/12305

7.Rodrigues MS, Santana LF, Graça EP, Gomes OV. Epidemiologia de traumatismo craniencefálico em um hospital. Rev Soc Bras Clin Med $2018 ; 16: 21-4$.

http://docs.bvsalud.org/biblioref/2018/06/884987/dezesseis1 vinteu m.pdf

8. Almeida LCF, Brasileiro ME. Atuação do Enfermeiro no Atendimento ao Paciente com Traumatismo Crânioencefálico: Revisão Bibliográfica. Rev Cien Multidiscipl Núcleo Conhecimento 2018;5:139-48.

https://www.nucleodoconhecimento.com.br/saude/paciente-comtraumatismo

9. Alcântara TFD, MARQUES IR. Avanços na Monitorização Neurológica Intensiva: implicações para a enfermagem. Rev. bras. enferm. Brasília , v. 62, n. 6, p. 894-900, dez. 2009.

https://doi.org/10.1590/S0034-71672009000600015

10. Chowdhury T, Kowalski S, Arabi Y, Dash HH. General intensive care for patients with traumatic brain injury: An update. Saudi J Anaesth 2014;8:256-63.

http://dx.doi.org/10.4103/1658-354X.130742

11.Talving P, Karamanos E, Teixeira PG, Skiada D, Lam L, Belzberg H, et al. Intracranial pressure monitoring in severe head injury: compliance with Brain Trauma Foundation guidelines and effect on outcomes:a prospective study. J Neurosurg 2013;119:1248-54. http://dx.doi.org/10.3171/2013.7.JNS122255

12. Carney N, Totten AM, O'Reilly C, Ullman JS, Hawryluk GWJ, Bell MJ, et al. Guidelines for the Management of Severe Traumatic Brain Injury, Fourth Edition. Neurosurgery 2017;80:6-15. http://dx.doi.org/10.1227/NEU.0000000000001432

13. Reyaz AP, Arif HS, Mohammad AS, Toufeeq AM, Abdul WM, Suhail $S$, et al. Our experience with management and outcome of isolated traumatic brain injury patients admitted in Intensive. J Emerg Trauma Shock 2018;11:288-92. http://dx. doi.org/10.4103/JETS.JETS 3417 14.Correio RAPPV, Vargas MAO, Carmagnani MIS, Ferreira ML, Luaz KR. Desvelando competências do enfermeiro de terapia intensiva. Enfer FOCO 2015;6:46-50. https://doi.org/10.21675/2357707X.2015.v6.n1/4.576

15. Wijdicks EF, Menon DK, Smith M. Ten things you need to know to practice neurological critical care. Intensive Care Med 2014;41:318. http://dx.doi.org/10.1007/s00134-014-3544-9

16. Dash $\mathrm{HH}$, Chavali S. Management of traumatic brain injury patients. Korean J Anesthesiol 2018;71:12-21.

http://dx.doi.org/10.4097/kjae.2018.71.1.12 
17.Rosa NM, Lima JF, Inoue KC. Conhecimento da equipe de enfermagem sobre neurointensivismo e a influência da educação contínua. Cienc Cuidado Saúde 2013;12. https://doi.org/10.4025/cienccuidsaude.v12i1.15031

18.Damkliang J, Considine J, Kent B, Street M. Using an evidencebased care bundle to improve Thai emergency nurses' knowledge of care for patients with severe traumatic brain injury. Nurse Educ Pract 2015;15:284-92. https://doi.org/10.1016/j.nepr.2015.03.007

19.Varghese R, Chakrabarty J, Menon G. Nursing Management of Adults with Severe Traumatic Brain Injury: A Narrative Review. Indian J Critical Care Med 2017;21:684-97.

http://dx.doi.org/10.4103/ijccm.IJCCM 23317

20.Joseph D, Marek C, Hadie A, Danilo C, Angelos GK, Frederick AZ, et al. Twenty-Five Years of Intracranial Pressure Monitoring After Severe Traumatic Brain Injury: A Retrospective, Single-Center Analysis. Neurosurgery 2018;85:E75-82.

http://dx.doi.org/10.1093/heuros/nyy468

21.Stocchetti N, Maas AIR. Traumatic Intracranial Hypertension. New Engl J Med 2014;370:2121-30.

http://dx.doi.org/10.1056/NEJMra1208708

22.Paranhos E. Traumatismo Crânio Encefálico. In: Guimarães HP, Assunção MSC, Carvalho FB, Japiassu AM, Veras KN, Nácul FE, et al. Manual de Medicina Intensiva: AMIB. São Paulo: Atheneu, 2014, p89104.

23. Diccine S, Osis S. Paciente neurológico: conceito e cuidados. In: Viana RAP, Torre M. Enfermagem em Terapia intensiva: Práticas Integrativas. Barueri: Manole; 2017, p790-806.

24.Conselho Federal de Enfermagem - COFEN (endereço na internet). Resolução No 0557/2017. Normatiza a atuação da equipe de enfermagem no procedimento de Aspiração de Vias Aéreas. Brasília: Conselho Federal de Enfermagem (acessado em 2020). Disponível em: http://www.cofen.gov.br/wp-content/uploads/2017/09/RES.-5572017.pdf.

25.Ribeiro CJ, Araújo AC, Brito SB, Dantas DV, Nunes MS, Alves JA, et al. Avaliação da dor de vítimas de traumatismo craniencefálico pela versão brasileira da Behavioral Pain Scale. Rev Bras Ter Intensiva 2018;30:42-9. http://dx.doi.org/10.5935/0103-507X.20180009

26.Yokobori S, Yokota H. Targeted temperature management in traumatic brain injury. J Inten Care 2016;4:28. http://dx.doi.org/10.1186/s40560-016-0137-4

27.Salgado PO, Silva LCR, Silva PMA, Chianca TCM. Métodos físicos para tratamento de febre em pacientes críticos: ensaio clínico controlado randomizado. Rev Esc Enfem USP 2016;50:823-30. http://dx.doi.org/10.1590/S0080623420160000600016

28. Conselho Federal de Enfermagem - COFEN (endereço na Internet). Resolução No 390/2011. Normatiza a execução, pelo enfermeiro, da punção arterial tanto para fins de gasometria como para monitorização de pressão arterial invasiva. Brasília: Conselho Federal de Enfermagem 
(acessado em 2020). Disponível em:

http://www.cofen.gov.br/resoluo-cofen-n-3902011 8037.html

29. Gonçalves FAF, Oliveira GF, Suzuki K, Miquelin B. Monitorização da Oxigenação e perfusão. In: Viana RAP, Torre M. Enfermagem em Terapia intensiva: Práticas integrativas. Barueri: Manole; 2017, p5019.

30.Mendes OLAM, SoareS YRC, Noleto LC, FontinelE AVC, Galvão MPS, Souza JM. Assistência de enfermagem em pacientes vítimas de traumatismo crânio encefálico: revisão integrativa. Rev Uningá 2018;55:33-46.

http://revista.uninga.br/index.php/uninga/article/view/2090.

31.Allan PA. Sistematização da Assistência de enfermagem(SAE). In: Viana RA, Torre M. Enfermagem em Terapia intensiva: praticas integrativa. Barueri: Manole; 2017, p150-157. 